from pleura cancer and melanoma. This cohort study is the most informative ever conducted in France among nuclear workers.

\section{THE PESTIMAT PROGRAM: DEVELOPMENT OF A CROP EXPOSURE MATRIX FOR PESTICIDE EXPOSURE ASSESSMENT IN AGRICULTURE}

1,2 Isabelle Baldi, ${ }^{1,2}$ Camille Carles, ${ }^{1}$ Anne Cantagrel, ${ }^{3,4}$ Yannick Lecluse, ${ }^{3,4}$ Elodie Niez, ${ }^{5}$ Pascale Fabbro-Peray, ${ }^{3,4}$ Pierre Lebailly. ${ }^{1}$ Univ. Bordeaux, ISPED, Laboratoire Santé Travail Environnement, Centre INSERM U 897 Epidemiologie-Biostatistique, F-33000 Bordeaux, France; ${ }^{2} \mathrm{CHU}$ de Bordeaux, Service de Médecine Du Travail, F-33000 Bordeaux, France; ${ }^{3}$ INSERM, UMR 1086- Cancers Et Préventions, F-14000 Caen, France; ${ }^{4}$ Centre François Baclesse, F-14000 Caen, France; ${ }^{5}$ DIM, Hôpital G Doumergue, F-34000 Montpellier, France

\subsection{6/oemed-2014-102362.108}

Objectives Pesticides include $\sim 1000$ chemicals with various toxicological properties. Pesticide exposure assessment represents a crucial methodological issue for epidemiological studies. A reconstitution of pesticides used in entire professional careers based only on the memory is questionable. PESTIMAT is a crop exposure matrix, which intends to reconstitute parameters of pesticide exposure in France.

Method PESTIMAT is made of tables crossing crops and active ingredients (a.i.) by year from 1950 to 2010 and providing the following exposure parameters: 1) probability corresponding to the proportion of farmers having used the a.i. (in\%), 2) frequency expressed as the number of treatment days, 3) intensity documented by the application rate of the a.i. (in $\mathrm{kg} / \mathrm{ha}$ ). Parameters were obtained by the combination of six complementary sources: i) registrations and withdrawals from the Agriculture Ministry, ii) information from ACTA* on products marketed iii) agricultural recommendations by the Plant Health Protection body, iv) treatment calendars provided by farmers, v) information from professionnal associations of farmers, vi) data from the industry

Results To date, 529 a.i. usable between 1950 and 2010 on 4 crops are included in PESTIMAT, corresponding to 160 fungicides (30\%), 160 herbicides (30\%) and 209 insecticides (40\%). The total numbers were comparable on wheat/barley $(\mathrm{N}=293)$, vineyards $(\mathrm{N}=280)$, and apple-growing $(\mathrm{N}=267)$ but lower on corn $(\mathrm{N}=196)$. We will present an illustration with data obtained for dithiocarbamates fungicides, and examples of use in epidemiological studies, e.g. PHYTONER and AGRICAN cohorts.

Conclusions The multisource information available in the PESTIMAT matrix should enable to assess associations between pesticides and health at the a.i. level.

\section{A CROSS-SECTIONAL STUDY OF MARKERS OF EARLY IMMUNOLOGICAL AND CARDIOVASCULAR HEALTH EFFECTS AMONG A POPULATION EXPOSED TO CARBON NANOTUBES: THE CANTES STUDY}

\begin{abstract}
${ }^{1}$ Roel Vermeulen, ${ }^{2}$ Anjoeka Pronk, ${ }^{1}$ Jelle Vlaanderen, ${ }^{3}$ Dean Hosgood, ${ }^{4}$ Nat Rothman, ${ }^{4}$ Allan Hildesheim, ${ }^{4}$ Debra Silverman, ${ }^{1}$ Annemarie Melis, ${ }^{2}$ Suzanne Spaan, ${ }^{2}$ Eef Voogd, ${ }^{5}$ Peter Hoet, ${ }^{5}$ Lode Godderis, ${ }^{4}$ Qing Lan. ${ }^{1} / R A S$ Utrecht University, Utrecht, The Netherlands; ${ }^{2}$ TNO, Zeist, The Netherlands; ${ }^{3}$ Albert Einstein College of Medicine, New York, NY, USA; ${ }^{4}$ National Cancer Institute, Bethesda, MD, USA; ${ }^{5}$ University of Leuven, Leuven, Belgium
\end{abstract}

10.1136/oemed-2014-102362.109
Objectives To assess the association between occupational exposure to carbon nanotubes (CNTs) and early immunological and cardiovascular health effects.

Method We collected biological samples (blood, urine, buccal and nasal epithelium cells) from a group of workers in a CNT production factory and approximately age and sex matched unexposed individuals working in a different workplace. Based on an initial assessment of exposure data collected in the weeks before biological sampling we further classified CNT production workers as operators (high probability of exposure) and R\&D workers (lower probability of exposure). We analysed blood samples for a panel of inflammatory markers.

Results We included 8 operators (median age 34.8), 16 R\&D workers (median age 32.2), and 43 controls (median age 30.6). Preliminary analyses provide some evidence for an association between CNT exposure and selected cytokines. We observed a similar pattern when we restricted our analyses to non-smoking men. Concentrations of cytokines were exposure dependently upregulated with higher levels among operators than R\&D workers; both higher than unexposed workers ( $\mathrm{p}$ for trend $<0.05$ ).

Conclusions We observed some indications of early immunological health effects in a pilot study conducted among workers exposed to CNTs. Further analyses are planned, including assessment of lung function, heart rate variability, oxidative stress, and blood coagulation markers. Extensive exposure measurements were conducted in the CNT production factory as well and additional analyses will use these individual exposure measurements to more thoroughly explore exposure dependent effects.

\section{DEVELOPMENT OF A SOURCE-BASED APPROACH TO ASSESSING OCCUPATIONAL EXPOSURE TO ELECTROMAGNETIC FIELDS IN THE INTEROCC STUDY}

${ }^{1}$ Javier Vila, ${ }^{2}$ Joseph D Bowman, ${ }^{3}$ Laurel Kincl, ${ }^{2}$ Dave L Conover, ${ }^{4}$ Martie van Tongeren, ${ }^{1}$ Jordi Figuerola, ${ }^{5}$ Lesley Richardson, ${ }^{1}$ Elisabeth Cardis, ${ }^{6}$ on behalf of the INTEROCC Study Group. ${ }^{1}$ Centre for Research in Environmental Health (CREAL), Barcelona, Spain; ${ }^{2}$ The National Institute for Occupational Safety and Health (NIOSH), Cincinnati, OH, USA; ${ }^{3}$ Oregon State University (OSU), Corvallis, OR, USA; ${ }^{4}$ Institute of Occupational Medicine (IOM), Edinburgh, UK; ${ }^{5}$ University of Montreal Hospital Research Centre (CRCHUM), Montreal, Canada; ${ }^{6}$ Institute of Occupational Medicine (IOM), Edinburgh, Spain

\subsection{6/oemed-2014-102362.110}

Objectives Exposure to electromagnetic fields (EMF) has become ubiquitous in modern life and concern has increased regarding possible associated health effects. To date, assessment of occupational exposure has relied on job-exposure matrices, with exposure estimates for very broad occupational categories. To move EMF research forward, a new approach was necessary. A source-based strategy, incorporating detailed information on tasks, equipment used and work organisation could allow a more individualised exposure assessment.

Method Information on occupational histories and sources of EMF was collected as part of the INTERPHONE-INTEROCC study, providing an opportunity to assess occupational EMF exposure by assigning exposure to each source used. A sourceexposure matrix (SEM) was developed based on measurements identified in the literature and estimates obtained through experts' elicitation, for sources without available measurements. This paper focuses on the SEM development methodology to ensure the quality and representativeness of the estimates. 
Results Estimates of exposure for 138 EMF sources were obtained from measurements (1424 aggregated records) extracted from 71 papers and hygiene reports (1974-2013). For each source, exposure was calculated by frequency band and dosimetry type, as the arithmetic and geometric means of all measurements identified. Standard deviations were included in order to characterise the variability of the estimates.

Conclusions A source-exposure matrix has been constructed for the most common sources of EMF in the workplace, based on the responses to the INTERPHONE-INTEROCC study questionnaire. This database currently represents the most comprehensive source of information on occupational EMF exposure and is available on request to researchers.

\section{OCCUPATIONAL USE OF INSECTICIDES, FUNGICIDES AND FUMIGANTS AND RISK OF NON-HODGKIN LYMPHOMA AND MULTIPLE MYELOMA IN THE AGRICULTURAL HEALTH STUDY} ${ }^{4} J o s e p h$ Barker, ${ }^{4}$ Dennis Buckman, ${ }^{5}$ Kent Thomas, ${ }^{6}$ Dale Sandler, ${ }^{7} J a n e$ Hoppin, ${ }^{1}$ Stella Koutros, 'Gabriella Andreotti, 'Jay Lubin, 'Aaron Blair, ${ }^{1}$ Laura Beane Freeman. ${ }^{1}$ Occupational and Environmental Epidemiology Branch, National Cancer Institute, Bethesda, MD, USA; ${ }^{2}$ University of lowa, Department of Epidemiology, lowa City, IA, USA; ${ }^{3}$ National Institute for Occupational Safety and Health, Cincinnati, OH, USA; ${ }^{4} I M S$, Calverton, MD, USA; ${ }^{5}$ National Exposure Research Laboratory, U. S. Environmental Protection Agency, Research Triangle, NC, USA; ${ }^{6}$ National Institute of Environmental Health Sciences, Research Triangle, NC, USA; ${ }^{7}$ North Carolina State University, Raleigh, NC, USA

\subsection{6/oemed-2014-102362.111}

Objectives Farming and exposure to pesticides have been linked to non-Hodgkin lymphoma (NHL), and multiple myeloma $(\mathrm{MM})$ in previous studies. We evaluated use of insecticides, fungicides and fumigants and risk of NHL, including MM and other NHL sub-types in the Agricultural Health Study, a US-based prospective cohort study.

Method A total of 527 cases occurred among 55875 pesticide applicators from enrollment (1993-1997) through 2011 in Iowa and 2010 in North Carolina. Information on pesticide use, other agricultural exposures and other factors was obtained from questionnaires at enrollment and follow-up approximately five years later (1999-2005). Information from these questionnaires was used to create lifetime-days and intensity-weighted lifetime-days of pesticide use. Poisson regression and polytomous logit models were used to calculate relative risks (RR) and 95\% confidence intervals (CI) to evaluate associations between 26 pesticides and NHL and five NHL-subtypes including multiple myeloma, while adjusting for potential confounding factors.

Results Statistically significant positive exposure-response trends occurred between overall NHL risk and lindane ( $\mathrm{p}$-trend = 0.004 ) and DDT ( $\mathrm{p}$-trend $=0.02$ ). In addition, ever use of terbufos was associated with NHL overall $(\mathrm{RR}=1.2 ; \mathrm{CI}=1.0-1.5)$, but with no exposure-response trend. In sub-type analyses, terbufos and DDT were associated with small cell lymphoma/ chronic lymphocytic leukaemia/marginal cell lymphoma. In addition, lindane and diazinon were associated with follicular lymphoma and permethrin with MM although tests of homogeneity did not show significant differences in exposure-response among NHL-subtypes for any chemical.

Conclusions These findings are among the first to suggest links between DDT, lindane, permethrin, diazinon and terbufos and specific NHL subtypes.

\section{GENE-SPECIFIC DNA METHYLATION AS A VALUABLE TOOL FOR RISK ASSESSMENT: THE CASE OF OCCUPATIONAL EXPOSURE TO DIFFERENT VOC'S IN MEXICAN WORKERS}

${ }^{1}$ Octavio Jiménez-Garza, ${ }^{2}$ Andrea Baccarelli, ${ }^{2}$ Hyang-Min Byun, ${ }^{3}$ Giovanni Battista Bartolucci, ${ }^{3}$ Mariella Carrieri. 'University of Gunajuato Campus León, Health Sciences Division, Léon, Guanajuato, Mexico; ${ }^{2}$ Harvard School of Public Health, Laboratory of Human Environmental Epigenomics, Boston, Massachusetts, USA; ${ }^{3}$ University of Padova, Department of Medicine, Padova, Italy

\subsection{6/oemed-2014-102362.112}

Objectives To determine gene-specific methylation levels (promoter region) on genes from critical cellular pathways in persons occupationally exposed to a single volatile organic compound (VOC) or to a mixture of them

Method Workers from two tanneries, two shoe factories, and two gas stations were included (exposed groups to VOC's). We also included administrative workers (reference group), all of them from the city of León Guanajuato, México. In tannery workers we measured individual exposure levels to three different VOC's; for the rest of the groups we measured up to 7 VOC's. After exposure characterisation, we took blood samples and extracted DNA in order to determine, by PCR-pyrosequencing, methylation levels in genes involved in inflammation, DNA repair, oxidative stress and xenobiotic metabolism pathways.

Results Only toluene environmental levels were higher in tannery workers. Workers from the leather shoe factory showed the highest exposure levels for up to five different VOC's. There was no statistical significant difference in gene-specific methylation for tannery workers when compared to the control group. For the leather shoe factory workers, we found hypermethylation in the TNFa, SOD1 and TOP2A promoter regions compared to the control group or to other exposed groups. Gas station attendants showed hypermethylation for the IL6 gene compared to controls Conclusions Occupational exposure to a mixture of VOC's has important effects on the methylation status of genes involved in inflammation, DNA repair and oxidative stress. These epigenetic changes, detectable at a pre-clinical stage, represent a valuable tool for performing an early risk assessment in these populations

\section{PREVENTING NEEDLESTICKS AND OTHER SHARPS INJURIES TO HOME CARE AIDES: RESULTS OF A SURVEY TO IDENTIFY HAZARDS DURING HOME VISITS}

${ }^{1}$ David Kriebel, ${ }^{1}$ Natalie Brouillette, 'Pia Markkanen, 'Catherine Galligan, 'Susan Sama, ${ }^{1}$ Rebecca Gore, ${ }^{2}$ Angela Laramie, 'Daniel Okyere, ${ }^{1}$ Chuan Sun, ${ }^{2}$ Letitia Davis, ${ }^{1}$ Margaret Quinn. 'University of Massachusetts Lowell, Lowell, MA, USA; ${ }^{2}$ Massachusetts Department of Public Health, Boston MA, USA

\subsection{6/oemed-2014-102362.113}

Objectives To follow up on our previous finding that home care (HC) aides have a substantial risk of injury with used sharp medical devices, this study's goal was to identify modifiable aspects of aide-client encounters during HC visits that increase risk of sharps injuries (SI).

Method A survey of 1249 HC aides was conducted in eastern Massachusetts. Approximately half the participants (634) were employed by HC agencies, and half (615) directly by HC clients and their families. A questionnaire gathered data on aides' most recent home visits, enabling quantification of hazardous working conditions. The specific investigation described here focused on understanding determinants of hazardous conditions likely to increase the risk of SI including: the aide finding used sharps 